\title{
AVALIAÇÃO AUDIOLÓGICA PRÉ-CIRURGIA OTOLÓGICA DE INDIVÍDUOS COM FISSURA LABIOPALATINA OPERADA
}

\author{
Pre-surgical auditory assessment of subjects \\ with operated cleft lip and palate
}

\author{
Francine Raquel dos Santos ${ }^{(1)}$, Silvia Helena Alvarez Piazentin-Penna ${ }^{(2)}$, Giovana Rinaldi Brandão ${ }^{(3)}$
}

\begin{abstract}
RESUMO
Objetivo: descrever as características audiológicas de indivíduos com fissura labiopalatina operada (FLP) e indicação de cirurgia otológica, comparando os grupos quanto ao tipo e grau da perda auditiva, bem como a curva timpanométrica. Método: análise de 150 prontuários, ambos os gêneros, idade igual ou superior a 4 anos, FLP e indicação de cirurgia otológica, divididos em 3 grupos: I Tubo de ventilação (TV), II - Timpanoplastia e III - Timpanomastoidectomia, analisando aspectos quanto a entrevista audiológica, audiometria tonal limiar e imitanciometria. Resultados: o grupo I apresentou porcentagem maior de cirurgia bilateral (86\%), o que não ocorreu nos demais grupos. $\mathrm{Na}$ entrevista audiológica, $83 \%$ apresentou algum tipo de queixa auditiva, sendo a mais frequente a perda auditiva (64\%) com $\mathrm{p}<0,05$ entre os grupos I e II; I e III. O tipo de perda auditiva de maior ocorrência foi condutivo bilateral (56\%) seguido de unilateral (35\%), com $p<0,05$ entre os grupos I e II; I e III. A perda de grau leve unilateral foi a de maior ocorrência (41\%), seguida de grau leve a moderada bilateral (20\%), com $p<0,05$ entre os três grupos. A curva timpanométrica mais frequente foi a do tipo B bilateral (39\%) com $\mathrm{p}<0,05$ entre os três grupos. Conclusão: a maioria dos indivíduos apresentou algum tipo de queixa na entrevista audiológica e alterações na audiometria tonal limiar e imitanciometria. A maioria dessas alterações foi compatível com problemas de orelha média, com perda auditiva do tipo condutiva, de grau leve e bilateral, independentemente da indicação cirúrgica.
\end{abstract}

DESCRITORES: Fissura Palatina; Fenda Labial; Otite Média; Audiologia

\section{INTRODUÇÃO}

A fissura labiopalatina é uma anomalia congênita que compromete o terço médio da face, especificamente a maxila, sendo caracterizada pela falta de fusão dos processos faciais, que tem início a partir da $4^{a}$ semana de vida intra-uterina ${ }^{1}$,

(1) Fonoaudióloga; Aluna do Curso de Aprimoramento do Hospital de Reabilitação de Anomalias Craniofaciais da Universidade de São Paulo, HRAC-USP, Bauru, SP, Brasil.

(2) Fonoaudióloga; Diretora Técnica dos Serviços Complementares do Hospital de Reabilitação de Anomalias Craniofaciais da Universidade de São Paulo, HRAC-USP, Bauru, SP, Brasil; Doutora em Distúrbios da Comunicação Humana.

(3) Fonoaudióloga do Hospital de Reabilitação de Anomalias Craniofaciais da Universidade de São Paulo, HRAC-USP, Bauru, SP, Brasil; Mestre em Distúrbios da Comunicação Humana.

Conflito de interesses: inexistente podendo causar comprometimentos de diversas ordens, como as alterações estéticas, emocionais e funcionais, requerendo a atuação de uma equipe interdisciplinar.

Dentre os comprometimentos funcionais podese destacar as alterações auditivas, em que grande parte da literatura é unânime em afirmar que a fissura labiopalatina acarreta problemas audiológicos e otológicos ${ }^{2-9}$. A maior ocorrência destes problemas acomete a orelha média, acarretando disfunção tubária, otite média com efusão e consequente perda auditiva, sendo a do tipo condutivo, de grau leve e bilateral a mais comumente encontrada ${ }^{10-13}$, com medida de imitância acústica revelando ausência do pico indicativo de pressão ${ }^{14}$, na presença de efusão na orelha média ${ }^{15}$.

A efusão da orelha média tem sido apontada como um fator desencadeante deste tipo de perda auditiva em indivíduos com fissura labiopalatina, em virtude da malformação anatômica e da defi- 
ciência funcional da musculatura da tuba auditiva com aquela do palato mole ${ }^{3,4}$. Além dessas alterações, também podem ser encontradas alterações morfológicas da tuba auditiva, como a hipoplasia ou displasia do tórus tubário e anormalidades nas cartilagens tubárias ${ }^{4,10}$.

Sabe-se que para o funcionamento adequado da orelha média é imprescindível que a tuba auditiva desempenhe corretamente as suas funções de drenagem, aeração e proteção da orelha média frente às secreções da nasofaringe. Em condições normais o óstio faríngeo da tuba auditiva permanece fechado, dilatando-se durante a deglutição, bocejo ou quando elevada pressão é produzida na nasofaringe. $\mathrm{Na}$ fissura palatina, ocorre uma deficiência na ação do músculo tensor do véu palatino na abertura da tuba auditiva, pois sem a inserção apropriada dos músculos do palato (principalmente a do tensor do véu palatino) não seria possível a dilatação do óstio e do lúmem tubário ${ }^{16}$.

Alguns autores ${ }^{17}$ salientaram a importância do papel da tuba auditiva na ventilação e proteção da cavidade da orelha média e afirmaram que a imaturidade da cartilagem da tuba auditiva em crianças com fissura palatina, especialmente, da lâmina lateral, pode ser um significante fator de risco para o desenvolvimento da otite média com efusão.

Na criança a tuba auditiva é mais curta e horizontalizada em relação à parte nasal da faringe e à oreIha média. Assim, ocorre facilitação para a entrada de substâncias estranhas, tal como a secreção, tornando-a mais suscetível às infecções de orelha média, contribuindo dessa forma para a instalação de perda auditiva. Com o crescimento, a tuba auditiva torna-se mais longa e verticalizada, protegendo melhor a orelha média de infecções ${ }^{15,18}$.

Qualquer tipo de perda auditiva pode trazer sérios efeitos no desenvolvimento da criança. A otite média crônica durante o período crítico do desenvolvimento de linguagem pode resultar na produção e percepção alteradas da fala ${ }^{19}$, sendo fundamental o diagnóstico e o tratamento adequado da mesma 11,13, evitando-se a instalação de uma perda auditiva e o possível comprometimento do desenvolvimento linguístico, cognitivo, escolar e interpessoal.

De acordo com o Joint Committee on Infant Hearing ${ }^{20}$, dentre os indicadores de risco para a perda auditiva destacam-se as anomalias craniofaciais. Sendo assim, indivíduos com fissura labiopalatina são considerados de risco sob o aspecto auditivo, devendo ser avaliados e acompanhados por fonoaudiólogos e otorrinolaringologistas, buscando a saúde auditiva dessa população.

A literatura mostra que mesmo após a correção cirúrgica do palato as alterações da orelha média podem persistir ${ }^{21}$. Devido aos frequentes episódios de otite média que acometem os indivíduos com fissura labiopalatina e suas prováveis sequelas, cirurgias otológicas como inserção de tubo de ventilação, timpanoplastia e timpanomastoidectomia, associadas ou não ao tratamento medicamentoso, fazem parte do tratamento ${ }^{22}$.

Estudos sugerem que a técnica de Furlow para a correção do palato pode influenciar no melhor funcionamento da tuba auditiva, bem como na redução de indicação de tubo de ventilação ${ }^{23}$.

Apesar da microcirurgia otológica com inserção de tubo de ventilação na membrana timpânica ter se tornada modalidade padrão de tratamento para crianças com otite média secretora, com ou sem fissura de palato, a literatura mostra divergência de opiniões no planejamento do tratamento quanto à necessidade e época ideal para a sua realização ${ }^{24}$.

O tratamento conservador da otite média com efusão em crianças com fissura labiopalatina demonstrou ser mais seguro e sem efeitos adversos em longo prazo. Apenas deveriam ser inseridos tubos de ventilação quando existisse perda de audição e efusão persistente na orelha média por mais de 3 meses ${ }^{8,25}$.

Em um estudo prospectivo para investigar os achados otológicos, audiológicos e radiológicos em crianças com fissura palatina com quadro de otite média com efusão, tratadas na idade de seis meses com inserção de tubo de ventilação bilateralmente, os autores sugerem que o prognóstico de crianças com fissura submetidas à timpanostomia precocemente é favorável. $O$ tratamento ativo garante audição normal durante os anos críticos de desenvolvimento da fala e linguagem e mantém o desenvolvimento da mastoide sob boa aeração ${ }^{18}$.

Em contrapartida, estudos sugerem que pacientes com fissura palatina devem ter intenso e detaIhado acompanhamento otológico e que se faz necessário maiores pesquisas para determinar o tratamento agressivo com tubos de ventilação ${ }^{10}$, sendo recomendada a realização de avaliação otológica rigorosa aos pacientes que necessitam do uso de tubos de ventilação em longo prazo, devido às possíveis complicações da cirurgia, principalmente o colesteatoma ${ }^{26,27}$, tornando-se importante o diagnóstico precoce em estágios prévios a formação deste problema ${ }^{28}$.

Quanto à mastoidectomia, foi descrito que a sua realização obedece a fatores relacionados ao processo da doença e as condições relacionadas ao próprio paciente. Este procedimento pode ser realizado em técnica com cavidade fechada, também chamada de simples ou conservadora, em que o canal auditivo externo é deixado intacto e com cavidade aberta em que a parede posterior do canal 
auditivo externo é aberta e a cavidade mastoide se comunica livremente com o meio ambiente via canal auditivo externo, ou seja, promove-se a exteriorização da mastoide e/ou cavidade timpânica. $\mathrm{Na}$ mastoidectomia radical, as estruturas da oreIha média (tímpano e ossículos) são removidas e promove-se a exteriorização da cavidade timpânica sem reconstrução funcional. Quando os ossículos são preservados, o procedimento também é chamado de mastoidectomia radical modificada ${ }^{29}$. Em indivíduos com fissura labiopalatina, o colesteatoma é muito prevalente e a escolha da técnica cirúrgica é complexa. Como não existe tratamento clínico para o colesteatoma, a intervenção é sempre cirúrgica e a escolha da técnica baseia-se no propósito de erradicar o colesteatoma em um único tempo, frente a um possível abandono do tratamento por parte do paciente, que é muito frequente. A escoIha da técnica fechada, em particular, depende de fatores anatômicos, funcionais e patológicos da tuba auditiva, e, principalmente, da possibilidade de retornos periódicos para avaliação de recidivas da doença infecciosa ${ }^{22}$.

Dessa forma, fica evidente a necessidade do acompanhamento de indivíduos com fissura labiopalatina por uma equipe interdisciplinar, com o intuito de diagnosticar as alterações auditivas precocemente, oferecer tratamento adequado (medicamentoso e/ou cirúrgico), evitando-se a instalação e/ou evolução da perda auditiva e, consequentemente, a privação sensorial auditiva.

Os objetivos deste estudo foram:

- Descrever as características audiológicas de indivíduos com fissura labiopalatina operada e indicação de cirurgia otológica, tais como: microcirurgia otológica com inserção de tubo de ventilação, timpanoplastia e timpanomastoidectomia.

- Comparar os tipos de cirurgias otológicas com os resultados da entrevista audiológica, audiometria tonal limiar e medidas de imitância acústica.

\section{MÉTODO}

\section{Constituição da amostra}

Foram analisados 150 prontuários de pacientes, sendo 119 (79\%) do gênero masculino e 31 (21\%) do gênero feminino, com idade igual ou superior a 4 anos, com fissura labiopalatina operada e com indicação de cirurgia otológica, regularmente matriculados no Hospital de Reabilitação de Anomalias Craniofaciais - HRAC/USP. Esses prontuários foram selecionados de forma aleatória a partir de uma listagem fornecida pelo Centro de Processamento de Dados do HRAC e foram divididos em 3 grupos, de acordo com o tipo de indicação cirúrgica:
GRUPO I: composto de 50 pacientes, que foram submetidos à avaliação audiológica no período de até 1 mês antes da realização da microcirurgia otológica com inserção de tubo de ventilação;

GRUPO II: composto de 50 pacientes, que foram submetidos à avaliação audiológica no período de até 1 mês antes da realização da timpanoplastia;

GRUPO III: composto de 50 pacientes, que foram submetidos à avaliação audiológica no período de até um mês antes da realização da timpanomastoidectomia.

\section{Procedimentos}

\subsection{Coleta de Dados}

Para a coleta das informações foi elaborado um protocolo específico contendo a identificação do paciente, data de nascimento, gênero, número de registro no hospital, tipo de fissura, cirurgia otológica realizada e informações referentes à documentação audiológica anteriormente a cirurgia otológica, quanto à entrevista audiológica, audiometria tonal limiar e medidas da imitância acústica para caracterizar o tipo e grau da perda auditiva.

- Entrevista Audiológica: foi realizada por fonoaudiólogos, com os pais ou responsáveis sob forma de perguntas dirigidas, com o objetivo de obter informações sobre a audição da criança;

- Audiometria Tonal Limiar: as informações deste exame estavam contidas nos prontuários, em um audiograma, no qual os fonoaudiólogos anotaram os resultados dos limiares audiométricos nas frequências de 250 à $8000 \mathrm{~Hz}$ por condução aérea e, de 500 à $4000 \mathrm{~Hz}$ por condução óssea, sendo os exames realizados em cabina acústica. Para este procedimento foram utilizados os audiômetros modelo Midimate 622 (Madsen), modelo AD 27 (Interacoustics) e TBN 85 (Madsen-Eletronics), com fones TDH-39P;

- Medidas de Imitância Acústica: quanto às condições da orelha média, foram anotadas as informações contidas no prontuário, em um timpanograma, como a timpanometria e medida da complacência estática. Os equipamentos utilizados foram modelo AZ7R (Interacoustics) e modelo GSI33 versão 2, MIDDLE-EAR ANALYZER, (Grason Stadler).

Foram excluídos deste estudo, os indivíduos que apresentaram fissura de lábio isolada, síndromes e/ ou comprometimentos neurológicos, identificados no prontuário dos mesmos.

\subsection{Análise dos Resultados e Estatística}

A partir da entrevista audiológica, os dados obtidos foram classificados em: sem queixa auditiva, quando não era referido problema em relação à audição ou com queixas, quando era referidas perda auditiva, otite, otorreia e otalgia. 
$\mathrm{Na}$ audiometria tonal limiar, os achados foram classificados quanto ao tipo de perda auditiva em condutiva, mista e sensorioneural ${ }^{30}$. Para perda auditiva condutiva foram considerados limiares tonais aéreos piores ou iguais a $25 \mathrm{dBNA}$ e limiares ósseos normais (até $15 \mathrm{dBNA}$ ), indicativo de problema na orelha média e/ou externa; para perda auditiva sensorioneural foram considerados limiares tonais aéreos e ósseos abaixo do limite considerado normal, 25 dBNA e 15 dBNA, respectivamente, não havendo presença de "gap" aéreo-ósseo (diferença de limiar aéreo-ósseo não excede $10 \mathrm{~dB}$ ), sugerindo um problema de mecanismo sensorial ou neural; na perda auditiva mista, há um envolvimento de um problema condutivo associado a um problema no mecanismo sensorioneural, refletindo em limiares audiométricos tonais aéreos e ósseos abaixo do limite de normalidade, havendo presença de "gap" aéreo-ósseo.

Quanto ao grau, as perdas auditivas foram classificadas em leve, leve/moderado, moderado, moderado/severo, severo, severo/profundo e profundo ${ }^{30}$. Foi considerado grau leve quando os limiares tonais audiométricos aéreos variavam de 25 a $40 \mathrm{dBNA}$; moderado quando apresentavam valores de 45 a 70 dBNA; severo, para os limiares na faixa de 75 a 90 dBNA e profundo, quando os limiares se mostraram piores ou iguais a 95 dBNA. Ainda, quando em uma mesma orelha, os limiares apresentaram graus diferentes nas frequências testadas, foi feita classificação do grau do melhor e do pior limiar encontrado na perda auditiva, como, por exemplo, leve/moderado.

As curvas timpanométricas foram classificadas em: curva tipo A, B, C, Ad e Ar. Os limiares de complacência acústica estática entre 0,3 a $1,3 \mathrm{ml}$ e a pressão da orelha média entre -100 daPa e +50 daPa foram considerados normais ${ }^{14}$.

Este estudo foi realizado após aprovação do Comitê de Ética em Pesquisa (CEP) do Hospital de Reabilitação de Anomalias Craniofaciais (HRAC) da Universidade de São Paulo (USP) sob o ofício $n^{\circ}$. 207/2007.
Os dados foram tabulados em banco de dados e submetidos à análise estatística utilizando o Teste Qui-quadrado, sendo aceitos como significantes os valores de $p<0,05$.

\section{RESULTADOS}

Foram analisados os resultados referentes à entrevista audiológica, à audiometria tonal limiar e às medidas de imitância acústica, todos de acordo com o tipo de cirurgia realizada.

\section{Entrevista Audiológica}

$\mathrm{Na}$ entrevista audiológica foram coletadas informações quanto à presença e ausência de queixa auditiva, bem como o tipo de queixa, quando presente. Dos 150 indivíduos estudados, 83\% ( $n=125)$ apresentaram algum tipo de queixa (atual e/ou pregressa). Foi encontrado maior número de queixas nos grupos II e III, quando comparados ao grupo I, sendo estas diferenças estatisticamente significantes entre os grupos I e II; I e III $(p=0,0124)$ (Tabela 1).

Dentre as queixas auditivas relatadas foram encontradas: perda auditiva, otite, otorréia e otalgia. A queixa de perda auditiva foi encontrada nos três grupos estudados, porém, no grupo I, $50 \%$ dos indivíduos apresentaram queixa de perda auditiva bilateral $(n=25)$, enquanto os indivíduos dos grupos II e III apresentaram $48(n=24)$ e $46 \%(n=23)$ respectivamente, queixa de perda auditiva unilateral. As queixas de otite e otorréia tiveram maior porcentagem unilateralmente nos indivíduos dos grupos II e III, sendo $46 \%$ e $64 \%$ relacionada à otite e $42 \%$ e $64 \%$ relacionada à otorreia, respectivamente. Já no grupo I, observamos $44 \%$ de queixa de otite e $32 \%$ queixa de otorreia. Para as queixas descritas anteriormente, foram encontradas diferenças estatisticamente significantes entre o grupo I e os demais $(p<0,001)$. A queixa menos relatada foi otalgia, estando presente em apenas $37 \%$ da amostra estudada.

Tabela 1 - Distribuição da presença ou ausência de queixa auditiva segundo o critério tipo de indicação cirúrgica

\begin{tabular}{ccccccc}
\hline \multirow{2}{*}{ Grupos } & \multicolumn{9}{c}{ Queixas auditivas } & \multirow{2}{*}{ TOTAL } & \multirow{2}{*}{$(\%)$} \\
\cline { 2 - 5 } & Ausente & $\mathbf{( \% )}$ & Presente & $\mathbf{( \% )}$ & & $(100)$ \\
Grupo I & 15 & $(30)$ & 35 & $(70)$ & 50 & $(100)$ \\
Grupo II & 5 & $(10)$ & 45 & $(90)$ & 50 & $(100)$ \\
Grupo III & 5 & $(10)$ & 45 & $(90)$ & 50 & $(100)$ \\
\hline TOTAL & 25 & $(17)$ & 125 & $(83)$ & 150 &
\end{tabular}




\section{Audiometria Tonal Limiar}

Com relação ao tipo de perda auditiva, nos grupos I e III, a maior ocorrência foi de perda auditiva condutiva bilateral ( $82 \%$ e $52 \%$ respectivamente), seguida de perda auditiva condutiva unilateral (16\% e $26 \%$ ) e perda auditiva mista bilateral (2\% e 10\%). Já no grupo II, a maior ocorrência foi de perda auditiva condutiva unilateral (48\%), seguida de perda auditiva condutiva bilateral (34\%) e perda auditiva mista bilateral (6\%). Foram encontradas diferenças estatisticamente significantes entre os grupos para a orelha direita $(p=0,001)$ e entre os grupos I e II, I e III $(p=0,0199)$ (Tabela 2).

Quanto ao grau da perda auditiva, no grupo I a maior ocorrência foi de perda auditiva leve bilateral e leve para moderada unilateral (32\%). Nos grupos II e III a maior ocorrência foi de perda auditiva leve para moderada unilateral (34\% e $24 \%$ respectivamente). Foram encontradas diferenças estatisticamente significantes entre os grupo I, II e III na oreIha direita $(p<0,0001)$ e os grupos I e II na orelha esquerda ( $p=0,0011)$ (Tabela 3$)$.

Dentre os indivíduos que apresentaram alteração no traçado audiométrico foi observado nos três grupos maior ocorrência de perda auditiva em frequências combinadas (90\%), dentre essas o maior comprometimento acometeu todas as faixas de frequências $(61 \%)$. Já as perdas auditivas em frequências isoladas foram encontradas em $10 \%$ dos indivíduos unilateralmente.

Tabela 2 - Distribuição conjunta dos tipos de perda auditiva bilateral segundo o tipo de cirurgia

\begin{tabular}{|c|c|c|c|c|c|c|c|c|c|}
\hline \multirow{2}{*}{ Grupos } & \multirow{2}{*}{ Tipo de perda auditiva OD } & \multicolumn{6}{|c|}{ Tipo de perda auditiva OE } & \multirow{2}{*}{ TOTAL } & \multirow{2}{*}{$(\%)$} \\
\hline & & Condutiva & $(\%)$ & Mista & $(\%)$ & Normal & $(\%)$ & & \\
\hline \multirow{4}{*}{ Grupo I } & Condutiva & 41 & (82) & & & 4 & $(8)$ & 45 & (90) \\
\hline & Mista & & & 1 & $(2)$ & & & 1 & (2) \\
\hline & Normal & 4 & $(8)$ & & & & & 4 & (8) \\
\hline & TOTAL & 45 & $(90)$ & 1 & $(2)$ & 4 & $(8)$ & 50 & $(100)$ \\
\hline \multirow{5}{*}{ Grupo II } & Condutiva & 17 & (34) & 1 & $(2)$ & 11 & (22) & 29 & (58) \\
\hline & Sensorial & & & 1 & $(2)$ & & & 1 & (2) \\
\hline & Mista & 1 & (2) & 3 & $(6)$ & 1 & (2) & 5 & (10) \\
\hline & Normal & 13 & $(26)$ & 2 & $(4)$ & & & 15 & $(30)$ \\
\hline & TOTAL & 31 & (62) & 7 & (14) & 12 & (24) & 50 & (100) \\
\hline \multirow{5}{*}{ Grupo III } & Condutiva & 26 & (52) & 1 & $(2)$ & 9 & (18) & 36 & (72) \\
\hline & Sensorial & & & 1 & (2) & & & 1 & (2) \\
\hline & Mista & 4 & (8) & 5 & (10) & & & 9 & (18) \\
\hline & Normal & 4 & (8) & & & & & 4 & $(8)$ \\
\hline & TOTAL & 34 & (68) & 7 & (14) & 9 & (18) & 50 & (100) \\
\hline
\end{tabular}

Tabela 3 - Distribuição conjunta dos graus de perda auditiva bilateral segundo o tipo de cirurgia

\begin{tabular}{|c|c|c|c|c|c|c|c|c|c|c|c|c|c|}
\hline \multirow{2}{*}{ Grupos } & \multirow{2}{*}{ Grau da perda auditiva OD- } & \multicolumn{10}{|c|}{ Grau da perda auditiva $O E$} & \multirow{2}{*}{ TOTAL } & \multirow{2}{*}{ (\%) } \\
\hline & & Leve & $(\%)$ & Leve a moderada & $(\%)$ & Moderada & (\%) & Severa & $(\%)$ & Normal & $(\%)$ & & \\
\hline \multirow{5}{*}{ Grupo I } & Leve & 16 & (32) & 10 & (20) & & & & & 3 & (6) & 29 & (58) \\
\hline & Leve a moderada & 6 & (12) & 9 & $(18)$ & & & & & 1 & (2) & 16 & (32) \\
\hline & Moderada & & & & & 1 & (2) & & & & & 1 & (2) \\
\hline & Normal & 4 & (8) & & & & & & & & & 4 & (8) \\
\hline & TOTAL & 26 & $(52)$ & 19 & (38) & 1 & (2) & & & 4 & (8) & 50 & (100) \\
\hline \multirow{5}{*}{ Grupo II } & Leve & 4 & $(8)$ & 1 & (2) & & & & & 7 & (14) & 12 & (24) \\
\hline & Leve a moderada & 2 & (4) & 12 & (24) & 3 & (6) & & & 5 & (10) & 22 & (44) \\
\hline & Moderada & 1 & (2) & & & & & & & & & 1 & (2) \\
\hline & Normal & 2 & (4) & 12 & $(24)$ & 1 & $(2)$ & & & & & 15 & $(30)$ \\
\hline & TOTAL & 9 & (18) & 25 & $(50)$ & 4 & (8) & & & 12 & (24) & 50 & (100) \\
\hline \multirow{6}{*}{ Grupo III } & Leve & 3 & (6) & 5 & (10) & 1 & (2) & & & 6 & (12) & 15 & $(30)$ \\
\hline & Leve a moderada & 7 & (14) & 9 & (18) & 2 & (4) & & & 2 & (4) & 20 & $(40)$ \\
\hline & Moderada & 4 & (8) & 2 & (4) & 1 & (2) & 1 & (2) & 1 & (2) & 9 & (18) \\
\hline & Severa & & & & & 1 & (2) & 1 & (2) & & & 2 & (4) \\
\hline & Normal & 3 & (6) & 1 & (2) & & & & & & & 4 & (8) \\
\hline & TOTAL & 17 & (34) & 17 & (34) & 5 & (10) & 2 & (4) & 9 & (18) & 50 & (100) \\
\hline
\end{tabular}


Tabela 4 - Distribuição conjunta da curva timpanométrica bilateral segundo o tipo de cirurgia

\begin{tabular}{|c|c|c|c|c|c|c|c|c|c|c|c|c|c|c|c|c|c|}
\hline \multirow{2}{*}{ Grupos } & \multirow{2}{*}{ Curva timpanométrica OD } & \multicolumn{14}{|c|}{ Curva timpanométrica $\mathrm{OE}$} & \multirow{2}{*}{ TOTAL } & \multirow{2}{*}{ (\%) } \\
\hline & & A & (\%) & B & (\%) & C & (\%) & As & (\%) & Ad & (\%) & Não vedou & (\%) & Não realizada & (\%) & & \\
\hline \multirow{5}{*}{ Grupo I } & $A$ & & & 2 & (4) & 1 & (2) & & & & & & & & & 3 & (6) \\
\hline & B & 4 & (8) & 31 & (62) & 4 & (8) & & & & & & & & & 39 & (78) \\
\hline & C & & & 4 & (8) & 2 & (4) & & & & & & & & & 6 & (12) \\
\hline & $\mathrm{Ad}$ & & & 1 & (2) & & & & & 1 & $(2)$ & & & & & 2 & (4) \\
\hline & TOTAL & 4 & (8) & 38 & (76) & 7 & (14) & & & 1 & (2) & & & & & 50 & (100) \\
\hline \multirow{7}{*}{ Grupo II } & A & & & 6 & (12) & & & & & & & 4 & (8) & & & 10 & (20) \\
\hline & B & 2 & (4) & 8 & (16) & 1 & (2) & & & 1 & (2) & 2 & (4) & & & 14 & (28) \\
\hline & C & 1 & (2) & 4 & (8) & 1 & $(2)$ & & & & & 3 & (6) & & & 9 & (18) \\
\hline & As & & & 1 & (2) & & & & & & & & & & & 1 & (2) \\
\hline & $\mathrm{Ad}$ & & & 1 & (2) & & & & & & & & & & & 1 & (2) \\
\hline & Não vedou & 8 & (16) & 4 & (8) & 1 & (2) & 1 & (2) & 1 & (2) & & & & & 15 & (30) \\
\hline & TOTAL & 11 & (22) & 24 & (48) & 3 & (6) & 1 & (2) & 2 & (4) & 9 & (18) & & & 50 & (100) \\
\hline \multirow{6}{*}{ Grupo III } & $A$ & & & 1 & (2) & 1 & (2) & & & & & & & & & 2 & (4) \\
\hline & B & 5 & (10) & 19 & (38) & & & 1 & (2) & & & & & 2 & (4) & 27 & (54) \\
\hline & C & & & 2 & (4) & & & & & & & & & 1 & (2) & 3 & (6) \\
\hline & $\mathrm{Ad}$ & & & 1 & (2) & & & & & & & & & 2 & (4) & 3 & (6) \\
\hline & Não realizada & 4 & (8) & 2 & (4) & & & & & 2 & (4) & & & 7 & (14) & 15 & (30) \\
\hline & TOTAL & 9 & (18) & 25 & (50) & 1 & (2) & 1 & (2) & 2 & (4) & & & 12 & (24) & 50 & (100) \\
\hline
\end{tabular}

\section{Medidas de Imitância Acústica}

$\mathrm{Na}$ Tabela 4, observa-se que em todos os grupos estudados, a curva do tipo B (uni ou bilateral) foi a mais encontrada. Dentre as curvas alteradas, nos grupos I e III a maior ocorrência foi de curva tipo B bilateral $(62 \%$ e $38 \%)$, seguida de B unilateral $(30 \%$ e $28 \%$ ). No grupo II a maior ocorrência foi de curva tipo B unilateral (44\%), seguida de B bilateral (16\%). Foram encontradas diferenças estatisticamente significantes entre o grupo II e os demais $(p<0,001)$, devido à porcentagem de orelhas que não foram possíveis vedar.

\section{DISCUSSÃO}

A otite média crônica com efusão é uma importante doença, sendo definida como uma inflamação sem dor com efusão persistente na orelha média ${ }^{26}$. Este tipo de otite é considerado o mais prevalente em indivíduos com fissura de palato. Tais processos infecciosos são comuns nessa população por causa do mau funcionamento da tuba auditiva, decorrente da incompetência do músculo tensor do véu palatino ${ }^{3,4}$. Embora o músculo tensor do véu possa melhorar após o fechamento cirúrgico da fissura palatina, dificilmente é normalizado, e os episódios de otites podem tornar-se constantes ${ }^{21}$.

O efeito inicial da disfunção tubária é a pressão negativa da orelha média em decorrência do acúmulo de secreção nessa estrutura ${ }^{15}$. Uma disfunção da tuba auditiva persistente também pode ser a causa primária de um grande número de alterações sérias da orelha média, como atelectasia, perfuração timpânica e colesteatoma, observado principal- mente em indivíduos com fissura labiopalatina ${ }^{26}$, e a perda auditiva condutiva é o sintoma mais importante ${ }^{18}$.

Deve-se levar em consideração que, nos casos de perdas auditivas leves, os sintomas podem ser discretos, a ponto de não serem notados pelo indivíduo e/ou por seus familiares ${ }^{3}$ como, também, poderão apresentar alterações auditivas assintomáticas, ressaltando a importância do diagnóstico auditivo precoce, permitindo um tratamento adequado e evitando, muitas vezes, que uma deficiência auditiva se instale privando a criança de informações sensoriais e prejudicando o desenvolvimento de fala, linguagem e aprendizagem.

A respeito do tipo de queixa auditiva, de forma geral, a literatura limita-se a informar se a queixa está presente ou ausente, não informando qual o tipo de queixa mais comum de ser encontrado nos indivíduos com fissura labiopalatina ${ }^{3}$.

No presente estudo observou-se que, a maior parte da casuística (83\%), apresentava algum tipo de queixa auditiva, sendo a mais frequente a queixa de presença de perda auditiva $(64 \%)$ uni ou bilateral. A presença de queixa de otite e otorréia (atual ou pregressa) apresentaram maior porcentagem nos grupos II e III. Esses achados são compatíveis com os resultados audiométricos e de medidas de imitância acústica, pois estão diretamente relacionadas com alterações da orelha média. Tais resultados estão em concordância com outros autores ${ }^{26}$ que encontraram $63 \%$ de queixa auditiva.

A queixa menos relatada foi otalgia, estando presente em apenas 37\% da amostra estudada. Uma vez que a otite média serosa não acarreta sensa- 
ção de dor, acaba tornando-se uma patologia dita "silenciosa", passando despercebida pelo indivíduo ou pela família ${ }^{2,16}$, justificando o fato dos indivíduos do grupo I apresentarem menos queixa auditiva que os demais grupos.

A análise dos traçados audiométricos revelou a presença de algum tipo de alteração uni ou bilateral, sendo a perda auditiva de grau leve unilateral a de maior ocorrência $(n=62)$, seguida do grau leve a moderada bilateral $(n=30)$ e leve a moderada unilateral $(n=28)$, corroborando com estudos que verificaram maior ocorrência de alteração auditiva de grau leve ${ }^{10,11,28}$.

Em concordância com estudos anteriores ${ }^{2,16}$, ao analisar-se a distribuição percentual dos indivíduos quanto ao tipo de frequência e os lados afetados, verifica-se que as perdas auditivas em frequências isoladas ocorreram menos frequentemente quando comparadas com as combinadas (90\%). Tal achado torna-se relevante, pois quanto maior o espectro de frequência da perda auditiva, maior será a privação sensorial e, consequentemente, o desenvolvimento de linguagem pode ser prejudicado ${ }^{2}$.

Com relação ao tipo de perda auditiva, verificase que a de maior frequência foi o tipo condutivo bilateral $(n=84)$, seguido do tipo condutivo unilateral $(n=52)$ e mista bilateral $(n=9)$. Na literatura verificase que outros autores também encontraram esses resultados $2,3,11,13$.

No presente trabalho, no grupo I a maior ocorrência foi de perda auditiva leve bilateral e leve para moderada unilateral, já nos grupos II e III a maior ocorrência foi de perda auditiva leve para moderada unilateral. Na literatura encontram-se relatos de alta ocorrência de perda auditiva na população com fissura labiopalatina. Em estudo realizado em 1988, verificou-se que $90 \%$ dos casos apresentaram perda auditiva condutiva de grau leve a moderado, relatando que em alguns casos existe escassa pneumatização da mastóide e as otites frequentes na infância podem provocar lesões sensorioneurais assim como sequelas otológicas interferindo na audição ${ }^{31}$. Outros autores também encontraram perda auditiva condutiva na maioria dos indivíduos com fissura labiopalatina pesquisados ${ }^{2,13,16,28}$.

A partir da análise da avaliação timpanométrica, verifica-se que a curva de maior frequência foi do tipo B bilateral $(n=58)$, seguida do tipo B unilateral $(n=51)$. Alguns casos dos grupos II e III apresentaram perfuração da membrana timpânica ou secreção na orelha média, impossibilitando a realização da timpanomonetria, porém estes resultados foram compatíveis com as queixas auditivas relatadas, bem como os limiares audiométricos obtidos. Alterações timpanométricas também foram relatadas por outros autores 2,10,11,16 ao estudarem indivíduos com fissura labiopalatina.

O tratamento da otite média serosa tem seu enfoque na correção da perda auditiva condutiva e, para essa finalidade, a literatura relata principalmente o tratamento cirúrgico ${ }^{18,28}$, como a microcirurgia otológica com inserção de tubo de ventilação e timpanomastoidectomia, dependendo da condição clínica. Uma vez superada a otite média com efusão e na presença de perfuração seca de membrana timpânica persistente, pode ser indicada a timpanoplastia.

A microcirurgia otológica consiste na miringotomia, drenagem da secreção e inserção de tubo de ventilação nessa incisão. Esse procedimento tende a equalizar a pressão da orelha média, promovendo a ventilação e a saída da efusão desse local, que tende promover o restabelecimento dos limiares auditivos desses indivíduos ${ }^{22}$. A facilidade do procedimento e o pronto benefício auditivo fizeram desse método o padrão no tratamento para otite média com efusão, como descrito em alguns trabalhos ${ }^{5,18}$. Entretanto, este tratamento não tem sido adotado universalmente, pois pode ocasionar sérias complicações da membrana timpânica, como o colesteatoma e uma perfuração persistente, quando o tubo de ventilação é colocado por longos períodos ${ }^{10,21,26}$.

$\mathrm{Na}$ instituição em que foi realizado o presente estudo, a inserção de tubo de ventilação é realizada quando o indivíduo apresenta após a palatoplastia efusão persistente na orelha média, com exceção para os casos de otite média aguda recorrente antes da palatoplastia ou retração timpanométrica que justifique a cirurgia.

O indivíduo com fissura de palato e alteração do funcionamento da tuba auditiva necessita de cuidado especial, sendo recomendada, em alguns centros, a inserção do tubo de ventilação já na primeira cirurgia para reparar a fissura, independentemente da presença ou não de otite média, podendo trazer alterações como descrito anteriormente. Dessa forma, é necessária a realização de outros estudos para se definir a real eficácia da inserção do tubo de ventilação associada às cirurgias primárias para corrigir a fissura.

A timpanoplastia é também um tratamento cirúrgico que contribui para a conservação e recuperação da audição, inclusive em indivíduos com fissura labiopalatina. Nestes, as causas mais comuns de perfuração da membrana timpânica são as infecções recorrentes, a otite serosa e a utilização repetida do tubo de ventilação, ou mesmo, a sua não utilização ${ }^{22}$. $\mathrm{Na}$ avaliação audiológica anteriormente à cirurgia, o achado audiométrico mais frequente é a perda auditiva condutiva com "gap" aéreo-ósseo 
em torno de $20 \mathrm{~dB}$; resultado acima desse valor é sugestivo de comprometimento da cadeia ossicular. No presente estudo encontram-se resultados audiométricos com perda auditiva e gap aéreo-ósseo semelhantes ao encontrado na literatura citada. A função da tuba auditiva também deve ser avaliada, pois a sua permeabilidade influencia no resultado final da cirurgia.

No Hospital de Reabilitação de Anomalias Craniofaciais - USP, a realização das mastoidectomias obedece a fatores relacionados ao processo da doença e a condições relacionadas ao próprio paciente, como descrito anteriormente.

De acordo com a literatura, a cirurgia otológica é uma alternativa para o tratamento de indivíduos com fissura labiopalatina operada ${ }^{22}$. Assim, sabendo-se da universalidade da presença de otite média com efusão em indivíduos com fissura labiopalatina, é necessário o acompanhamento por parte da equipe envolvida no tratamento desses indivíduos, evi- tando uma possível privação sensorial que pode desencadear importantes problemas com a aquisição de fala e linguagem, assim como no processo de reabilitação da fala, no desenvolvimento cognitivo e no convívio social em virtude, principalmente, da diminuição dos estímulos recebidos do meio.

\section{CONCLUSÃO}

A partir da análise dos dados obtidos da avaliação audiológica de 150 indivíduos com fissura labiopalatina operada e com indicação para cirurgia otológica, verifica-se que a maioria dos indivíduos apresentou algum tipo de queixa na entrevista audiológica e alterações na audiometria tonal limiar e imitanciometria. A maioria dessas alterações foi compatível com problemas de orelha média, com perda auditiva do tipo condutiva, de grau leve e bilateral, independentemente da indicação cirúrgica.

\begin{abstract}
Purpose: to describe the auditory characteristics of subjects with operated cleft lip-palate (CLP) referred to otologic surgery and compare groups regarding the type and degree of hearing loss and tympanometric curve. Method: analysis of 150 records, of both genders, about 4-year old, with CLP and referred to otologic surgery, were divided in 3 groups (1- Ventilation tube (VT), 2- Timpanoplasty 3- Timpanomastoidectomy). Aspects were analyzed according to an auditory interview, pure-tone test and immitance measures. Results: as for the surgical reference, VT showed higher prevalence of bilateral surgery (86\%), which has not occurred with the other groups. In auditory interview $83 \%$ of the subjects had complaints on hearing, especially hearing loss $-64 \%(p<0.05$ among groups 1 and 2 , 1 and 3). The most recurrent hearing loss type was bilateral conductive (56\%) followed by unilateral $(35 \%)(p<0.05$ among groups 1 and $2 ; 1$ and 3$), 41 \%$ had middle unilateral hearing loss and $20 \%$ had moderate bilateral loss, ( $p<0.05$ among the 3 groups). Bilateral type $B$ was the most frequent regarding the tympanometric curve (39\%) $-p<0.05$ among the three groups. Conclusion: most of the subjects showed some kind of alteration on the auditory interview, in pure tone test and immitance measures. Many of these alterations were compatible with the middle ear pathology, with middle bilateral conductive hearing loss, regardless of the surgical reference.
\end{abstract}

KEYWORDS: Cleft Palate; Cleft Lip; Otitis Media; Audiology 


\section{REFERÊNCIAS}

1. Silva Filho OG, Freitas JAS. Caracterização morfológica e origem embriológica. In: Trindade IE, Silva Filho, OG, organizadores. Fissuras labiopalatinas: uma abordagem interdisciplinar. Bauru: Santos; 2007. p. 17-49.

2. Brandão GR. Características audiológicas de indivíduos com sinais clínicos de Síndrome Velocardiofacial [dissertação]. Bauru (SP): Universidade de São Paulo; 2002.

3. Piazentin-Penna SH. Identificação auditiva em crianças de 3 a 12 meses de idade com fissura labiopalatina [tese]. Bauru (SP): Universidade de São Paulo; 2002.

4. Arnold WH, Nohadani N, Koch KH. Morphology of the auditory tube and palatal muscles in a case of bilateral cleft palate. Cleft Palate Craniofac J. 2005; 42(2):197-201.

5. Chu KMY, McPherson B. Audiological status of Chinese patients with cleft lip/palate. Cleft Palate Craniofac J. 2005; 42(3):280-5.

6. Smith W, Yung M. How we do it: laser reduction of peri-tubal adenoids in selected patients with otitis media with effusion. Clin Otolaryngol. 2006; 31(1):69-72. http://dx.doi. org/10.1111/j.1749-4486.2006.01126.x

7. Feniman MR, Daniel BT, De Vitto LPM, Lemos ICC, Lauris JRP. Reconhecimento verbal de lactentes com fissura labiopalatina com e sem história de indicadores de risco para a audição. Rev Bras Otorrinolaringol. 2008; 74(4):601-5.

8. Phua YS, Salkeld LJ, de Chalain TM. Middle ear disease in children with cleft palate: protocols for management. Int J Pediatr Otorhinolaryngol. 2009 Feb; 73(2):307-13.

9. Weckwerth $\mathrm{PH}$, de Magalhães Lopes CA, Duarte MA, Weckwerth AC, Martins CH, Neto DL, et al. Chronic suppurative otitis media in cleft palate: microorganism etiology and susceptibilities. Cleft Palate Craniofac J. 2009; 46(5):461-7.

10. Tuncbilek G, Özgur F, Belgin E. Audiologic and tympanometric findings in children with cleft lip and palate. Cleft Palate Craniofac J. 2003; 40(3):304-9.

11. D'Mello J, Kumar S. Audiological findings in cleft palate patients attending speech camp. Indian $\mathrm{J}$ Med Res. 2007; 125(6):777-82.

12. Luthra S, Singh S, Nagarkar AN, Mahajan JK. The role of audiological diagnostics in children with cleft lip \& palate (CLP). Int J Pediatr Otorhinolaryngol. 2009; 73(10):1365-7.

13. Flynn T, Möller C, Jönsson R, Lohmander A. The high prevalence of otitis media with effusion in children with cleft lip and palate as compared to children without clefts. Int J Pediatr Otorhinolaryngol. 2009; 73(10):1441-6.
14. Jerger J. Clinical experience with impedance audiometry. Arch Otolaryngol. 1970; 92(4):311-24.

15. Gudziol V, Mann WJ. [Chronic eustachian tube dysfunction and its sequelae in adult patients with cleft lip and palate]. HNO. 2006; 54(9):684-8.

16. Costa Filho OA, Piazentin SH. Aspectos otológicos. In: Altmann EB. Fissuras labiopalatinas. Carapicuíba: Pró-Fono; 1997. p. 485-98.

17. Takasaki K, Sando I, Balaban CD, Ishijima K. Postnatal development of eustachian tube cartilage. A study of normal and cleft palate cases. Int $\mathrm{J}$ Pediatr Otorhinolaryngol. 2000; 52(1):31-6.

18. Valtonen H, Dietz A, Qvarnberg Y. Long-term clinical, audiologic, and radiologic outcomes in palate cleft children treated with early tympanostomy for otitis media with effusion: a controlled prospective study. Laryngoscope. 2005; 115(8):1512-6.

19. Stockard-Pope JE. Auditory development and hearing evaluation in children. Adv Pediatr. 2001; 48:273-99.

20. Joint Committee on Infant Hearing. Year 2000 position statement: principles and guidelines for early hearing detection and intervention programs. Pediatrics. 2000; 106(4):798-817.

21. Shaw R, Richardson D, McMahon S. Conservative management of otitis media in cleft palate. J Cranio Maxillofac Surg. 2003; 31(5):316-20. http://dx.doi.org/10.1016/S1010-5182(03)00074-x

22. Piazentin-Penna SH, Jorge JC. Avaliação e tratamento dos distúrbios da audição. In: Trindade IE, Silva Filho OG, organizadores. Fissuras labiopalatinas: uma abordagem interdisciplinar. Bauru: Santos; 2007. p. 165-72.

23. Smith LK, Gubbels SP, MacArthur CJ, Milczuk $\mathrm{HA}$. The effect of the palatoplasty method on the frequency of ear tube placement. Arch Otolaryngol Head Neck Surg. 2008; 134(10):1085-9.

24. Ponduri S, Bradley R, Ellis PE, Brookes ST, Sandy JR, Ness AR. The management of otitis media with early routine insertion of grommets in children with cleft palate - a systematic review. Cleft Palate Craniofac J. 2009; 46(1):30-8.

25. Quintero Noa JL, García Martinez DA, Hernández Cordero MC, Valls Pérez O. Colesteatoma asociado a secuencia de labio y paladar hendidos. Rev Cuba Pediatr. [periódico online]. 2008 abr-jun [acesso em out 2009]; 80(2):[about 7 p.]. Disponível em: http:// scielo.sld.cu/pdf/ped/v80n2/ped13208.pdf

26. Sheahan P, Blayney AW, Sheahan JN, Early MJ. Sequelae of otitis media with effusion among children with cleft lip and/or cleft palate. Clin Otolaryngol Allied Sci. 2002; 27(6):494-500.

27. Paquot-Le Brun $C$, Babin $E$, Moreau S, Bequignon A. [Otologic sequels in velopalatine clefts. Analysis and management]. Rev Stomatol Chir Maxillofac. 2007; 108(4):357-68. 
28. Goudy S, Lott D, Canady J, Smith RJ. Conductive hearing loss and otopathology in cleft palate patients. Otolaryngol Head Neck Surg. 2006; 134(6):946-8.

29. Cruz OLM, Campos CAH. Cirurgia para a otite média crônica. Acta Otorrinolaringol. 2005; 23(1):33-8.
30. Jerger J, Jerger S. Measurement of hearing in adults. In: Paparella MM, Shumrick DA, organizadores. Otolaryngology 2. ed. Philadelphia: WB Saunders; 1980. p. 1226.

31. Silva AA. Complicações otológicas em crianças fissuradas. Arq Inst Penido Burnier. 1988; 30(2):115-8.

DOI: $10.1590 / S 1516-18462010005000028$

RECEBIDO EM: 21/10/2009

ACEITO EM: 11/12/2009

Endereço para correspondência:

Silvia Helena Alvarez Piazentin-Penna

Setor de Fonoaudiologia

Hospital de Reabilitação de Anomalias

Craniofaciais - USP

Rua Silvio Marchione, 3-20

Bauru - SP

CEP: $17043-900$

E-mail: piazenna@usp.br 